\title{
Análisis longitudinal del comportamiento reproductivo de una población Toba en transición ${ }^{\star}$
}

\author{
Norberto Lanza** \\ Claudia Valeggia ${ }^{* \star *}$
}

Las variaciones demográficas de una población están frecuentemente asociadas a cambios ambientales. Por lo tanto, las particularidades sociales, económicas y culturales de poblaciones indígenas producirían una gran variedad de regímenes demográficos. Si bien en América Latina, en general, y Argentina, en particular, se registró un importante descenso de la fecundidad, las poblaciones indígenas exhiben cambios demográficos que difieren de esta tendencia. Este trabajo analiza parámetros de fecundidad y sus determinantes de la población Toba Cacique Sombrero Negro del norte argentino, la cual atraviesa cambios en su estilo de vida. Se entrevistaron 336 mujeres calculando las tasas de fecundidad, la probabilidad de agrandamiento de la familia y la tasa de esterilidad primaria para las mujeres nacidas entre 1920 y 1966. Se estimó la edad materna al primer hijo, el intervalo intergénesico, la edad materna al último hijo. Los resultados indican que, respecto a las mujeres nacidas entre 1920 y 1945, las mujeres nacidas entre 1946 y 1966, muestran un incremento de la fecundidad, una disminución de la edad materna al primer hijo, un mayor ritmo reproductivo y un incremento de la edad materna al último hijo. Estos resultados sugieren cambios sociales, sanitarios, biológicos y económicos favorables que habrían determinado el incremento de la fecundidad para la cohorte más joven.

Palabras-claves: Toba. Fecundidad. Determinantes de la fecundidad. Transición demográfica.

\footnotetext{
* Los autores quieren agradecer a tres anónimos revisores quienes a partir de sus sugerencias han permitido mejorar sustancialmente la calidad de este trabajo. También agradecemos muy especialmente a los integrantes de la población Toba Cacique Sombrero Negro por su paciencia y amistad. En particular nuestro gratitud a los agentes sanitarios Rodolfo Seta Caínt, Adolfo Caín, Juan Larrea y Nicolás Larrea por facilitarnos parte de la información utilizada en este trabajo. También a Benito Tevez y al personal médico del centro de salud de Pozo de Masa quienes gentilmente nos posibilitaron acceder a los registros de nacimientos y defunción. Esta investigación se desarrolló con fondos otorgados por el CONICET (Argentina), la L. S. B. Leakey Foundation, la Wenner-Gren Foundation, the National Geographic Society, y el TRIO Award from the University of Pennsylvania.

** Consejo Nacional de Investigaciones Científicas y Técnicas (CONICET), Instituto de Investigaciones GeoHistóricas, Resistencia, Argentina (norbertolanza@yahoo.com.ar).

${ }^{* \star \star}$ Dept. de Antropología, Universidad de Pennsylvania, EEUU (valeggia@sas.upenn.edu).
} 


\section{Introducción}

Es ampliamente aceptado que las variaciones demográficas de una población son frecuentemente el resultado de cambios ambientales, principalmente sociales, económicos y culturales (ZAVALA DE COSÍO, 1992, p. 11). Esto implica que los cambios demográficos están determinados por el contexto de cada población. En consecuencia, diferentes situaciones sociales, económicas y culturales producirían una gran variedad de regímenes demográficos. Este concepto es clave para comprender, por ejemplo, la heterogeneidad que caracteriza la disminución de la fecundidad en América Latina (CHACKIEL, 2004, p. 9), llevando a plantear la necesidad de desarrollar diferentes modelos de acuerdo al país o sector social considerado (CHACKIEL; SCHOLNIK, 2004, p. 51).

Las particularidades ambientales cobrarían mayor importancia en el estudio de los procesos demográficos de poblaciones indígenas. Sistemas económicos no capitalistas, estructuras sociales de parentesco diferentes al "modelo occidental" pueden determinar diversos patrones de fecundidad (SKINNER, 1997, p. 53; BENTLEY et al., 1993, p. 269). Por ejemplo, Rodríguez Vignoli (2004, p. 93) muestra que, independientemente del nivel educativo de la madre o del desarrollo económico de la población, los Aymará de Bolivia presentan una menor probabilidad de tener una fecundidad alta en las primeras edades reproductivas que la población no indígena. Esto lleva al autor a proponer el efecto de las particularidades culturales de la etnia sobre su comportamiento reproductivo.

Las variaciones en el tiempo de los factores ambientales pueden ser también relevantes para una mejor compresión de las diferentes tendencias temporales de la fecundidad en estos grupos. Poblaciones indígenas en procesos de transición de su estilo de vida muestran cambios temporales diferentes o incluso opuestos de la fecundidad en relación al descenso general de América Latina. Por ejemplo, estudios realizados en poblaciones indígenas de Bolivia (DEL POPOLO et al., 2005, p. 131 ), Paraguay (MELIÀ, 1997, p. 411) y Xavante en Brasil (SOUZA et al., 2011) han mostrado claros indicios del inicio de una disminución temporal de la fecundidad. Sin embargo, la comparación de los censos realizados en Paraguay en el año 1992 (MELIÀ, 1997, p. 411) y 2002 (ARCE BORDÓN, 2005, p. 93) muestra un descenso de la mortalidad infantil, un importante determinante de la disminución de la fecundidad (BONGAARTS, 1978, p. 105), al mismo tiempo que se produce un incremento de la fecundidad para poblaciones Guaná, Avá Guaraní, Toba Máskoy y Maká. Incrementos similares, y en poblaciones comparables, han sido también obtenidos en poblaciones Aché de Paraguay (HILL; HURTADO, 1996, p. 561), Kamaiurá (PAGLIARO; JUNQUEIRA, 2007, p. 37) y Kaiabi (PAGLIARO, 2002, p. 194) de Brasil. Es decir, un análisis más minucioso de los cambios de la fecundidad revela no sólo retrasos en el inicio de la transición si no también tendencias diferentes en el tiempo.

\section{La disminución de la fecundidad en Argentina}

Argentina se ha caracterizado por su precocidad en la disminución de la fecundidad comenzando a registrarse a partir del último tercio del siglo XIX. Por ejemplo, ya en el año 
1960 Argentina registraba una fecundidad de 3,1 hijos, es decir cerca de un 50\% menor registrada a la del resto del continente (BAY; MACADAR, 2001, p. 41).

No obstante, la Argentina comparte un rasgo común con el resto de América Latina que es la gran diferenciación demográfica en el interior del país. Por ejemplo, parámetros demográficos estimados por Munilla y Goldztein (2005, p. 24) a partir del censo realizado en el año 2001 muestran que a nivel nacional la tasa global de fecundidad (TGF) de hogares indígenas es mayor a un $47 \%$ con respecto a los hogares no indígenas.

Los Toba constituye una de la etnia de mayor representación en el país (12\%) (Encuesta Complementaria de Pueblos Indígenas, ECPI) y en la provincia de Formosa representan casi el 33\% de la población indígena y el 2,5\% de la población total (BANCO MUNDIAL, 2010, p. 34). Los Toba comenzaron a cambiar su estilo de vida fundamentalmente desde comienzos del siglo XX a partir de la colonización militar, religiosa y económica, las cuales determinaron importantes cambios sociales, económicos y culturales (BRAUNSTEIN; MILLER, 1999, p. 88; GORDILLO, 2006, p. 320). Por tal motivo, se espera que estos procesos hayan afectado la dinámica demográfica de las poblaciones que integran dicha etnia. El objetivo de este trabajo es estimar y analizar las variaciones de la fecundidad y sus determinantes ${ }^{1}$ en mujeres nacidas entre los años 1920 y 1966 en la población rural Toba Cacique Sombrero Negro, ubicada en la provincia argentina de Formosa.

\section{Breve introducción etnográfica de los Toba - población de estudio}

Desde tiempos precolombinos los Toba se han caracterizado por hábitos nómadas o semi-nómadas (BRAUNSTEIN; MILLER, 1999, p. 88) y una economía basada en la pesca, la caza y la recolección (ARENAS, 2003, p. 562). Sin embargo, la restricción al acceso a las grandes extensiones de tierra que fueron entregadas a particulares y la parcial integración en el mercado laboral y de consumo, fueron los principales factores determinantes del proceso de sedentarización de estas comunidades. No obstante, todavía existen pequeñas comunidades rurales que mantienen algunos aspectos propios de su cultura, alimentación y economía (BRAUNSTEIN; MILLER, 1999, p. 88; VALEGGIA et al., 2004, p. 123; GORDILLO, 2006, p. 320). Esta investigación se realizó en la población rural Toba denominada Cacique Sombrero Negro, localizada en la provincia de Formosa, a 550 km. de la capital provincial (Figura 1).

\footnotetext{
${ }^{1}$ Análisis preliminares muestran que sólo el $1.35 \%$ de las mujeres entrevistadas reportaron haber usado algún método anticonceptivo moderno (datos no publicados). Además, trabajos anteriores de Lanza et al. (2011, p. 27) y Lanza y Valeggia (2013, p. 11, 2013a, p. 19) han evidenciado una elevada fecundidad de esta población. El conjunto de estos antecedentes sugieren un régimen de fecundidad natural. Por tal motivo, los autores adoptarán la definición y clasificación de determinantes de la fecundidad dada por Wood (1994) quien resalta aquellos de mayor ponderación sobre poblaciones con este tipo de comportamiento reproductivo. El autor define determinantes como aquellas variables intermedias biológicas y comportamentales a través de las cuales factores económicos, culturales y ambientales afectan la fecundidad.
} 
FIGURA 1

Ubicación geográfica de la población Toba Cacique Sombrero Negro

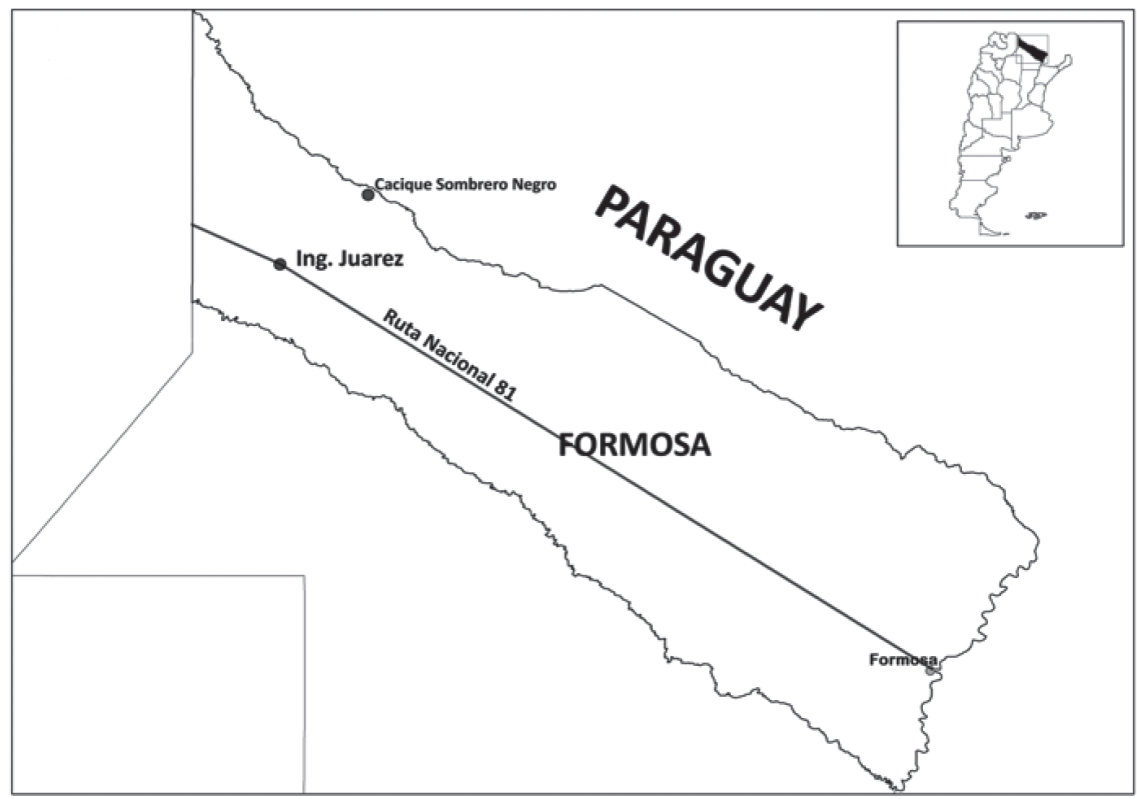

Fuente: Adaptado de Gordillo (2002, p. 262.).

En el año 2002, sus integrantes conformaban una población de 1739 personas, las cuales mantienen aspectos de su estilo de vida forrajeador (ARENAS, 2003, p. 562; VALEGGIA et al., 2004, p. 123). A pesar de esto, importantes cambios culturales, económicos y sociales se han producido vinculados a un creciente sedentarismo (BRAUNSTEIN; MILLER, 1999, p. 88; MENDOZA, 2002, p. 235). Desde comienzos del siglo XX hasta finales de la década de 1960, los Toba migraban en forma estacional a los ingenios azucareros; estas migraciones comprendían entre el 50\% y 75\% de la población y permitía vender su mano de obra y acceder, aunque en forma incipiente, acceso a la economía de mercado (GORDILLO, 1996, p. 135). El proceso de sedentarización de estas comunidades se aceleró a partir de comienzos de la re-instauración del regimen democrático en Argentina a finales del año 1983 debido principalmente al otorgamiento de 35.000 ha. por parte del gobierno provincial (MENDOZA, 2002, p. 235), al desarrollo de diferentes programas de asistencialismo y a un creciente número de aborígenes que trabajan para el gobierno provincial y municipal (GORDILLO, 2002, p. 16). De esta manera, aproximadamente en los últimos treinta años, esta población ha sufrido un proceso acelerado de cambio de su estilo de vida cazador-recolector hacia una creciente dependencia en la economía de mercado.

\section{Métodos}

\section{Fuentes de datos}

Con el fin de conocer las principales características de la vida reproductiva de las mujeres Toba, se realizaron entrevistas cerradas a mujeres mayores de 12 años de edad. La técnica 
empleada fue de muestreo de conveniencia. Se hizo un mapa de todos los asentamientos y se visitaron todas las casas habitadas, invitando a las mujeres que se encontraban en sus casas a participar. Se estima que aproximadamente 70\% de las mujeres mayores de 12 años que forman parte de esta población fueron entrevistadas, de las cuales 64 eran postmenopáusicas (alrededor del 60\% de las mujeres post-reproductivas). Ninguna mujer se negó a participar. La razón principal por la cual no se logró entrevistar a la totalidad de la población femenina mayor de 12 años fue que habían viajado al pueblo cercano (a $50 \mathrm{~km}$ ) para realizar trámites o para visitar a parientes. Se consideró que esto no representaba un riesgo de sesgo significativo porque las mujeres que no se encontraban presentes eran de variada edad y estado reproductivo. El trabajo de campo fue efectuado en los años 2002 y 2003, durante los cuales se realizó la mayor cantidad de entrevistas posibles. En el año 2006 se realizaron 12 entrevistas adicionales, pero principalmente se corroboró y actualizó la información recabada en los años 2002 y 2003.

Se relevó información de las mujeres como su nombre y apellido, fecha de nacimiento y edad de la menarca. Si la mujer tenía hijos, se le preguntaba por si habían nacido vivos o muertos, la fecha de nacimiento y el sexo de cada hijo. Para el caso de los hijos fallecidos, se interrogaba por la edad de su deceso y posible causa de su muerte. Para el caso de mujeres mayores de 45 años se preguntaba si continuaba menstruando regularmente al momento en que se realizó la entrevista para establecer su estado reproductivo.

Las entrevistas fueron realizadas en los hogares de las mujeres participantes y antes de comenzar se aseguró de obtener en forma oral el consentimiento informado de las mujeres. ${ }^{2}$ Luego de finalizadas, las fechas de nacimientos fueron verificadas a partir del Documento Nacional de Identidad (D.N.I.) y otras fuentes independientes como el Registro Civil, la sala médica y auxiliares de enfermería Toba ${ }^{3}$ a los fines de detectar posibles inconsistencias como la duplicación de información.

\section{Estimación de la fecundidad Toba}

A los fines de describir historias reproductivas completas, a partir del grupo de mujeres post-menopáusicas ( $n=64$ ) se estimó la tasa de fecundidad específica por edades (fx) y la TGF. Para caracterizar la estructura etaria de la fecundidad se calculó la edad media de la fecundidad (fx) y el aporte a la fecundidad total por grupos quinquenales de edad. También se calculó la tasa de esterilidad primaria como la proporción de mujeres que habiendo alcanzado la menopausia, no declararon haber tenido embarazos. Para el cálculo de la progresión de paridad, se utilizó el método de los promedios móviles por grupos quinquenales de edad.

\footnotetext{
${ }^{2}$ El protocolo del estudio fue aprobado por el comité de ética (IRB) de la Universidad de Pennsylvania, ya que el proyecto obtuvo financiamiento del Centro de Investigaciones en Envejecimiento de la Universidad de Pennsylvania, para lo cual se necesitaba aprobación del comité de ética institucional.

${ }^{3}$ Formosa fue pionera en desarrollar programas de salud que integraban indígenas al sistema médico-sanitario oficial. Sus funciones consistían asistir a los médicos, censar las comunidades, registrar los nacimientos y defunciones, reportar posibles casos de tuberculosis y Chagas, coordinar la distribución de leche para infantes y niños, controlar a las embarazadas y colaborar en las campañas de vacunación (BARGALLÓ, 1992, p. 128).
} 
Además, con el fin de registrar posibles cambios temporales de estos parámetros, dichas estimaciones se calcularon para las cohortes de mujeres nacidas entre los años 1920 y 1945 y para aquellas nacidas en el período 1946-66. Se comparó la edad media de la fecundidad entre períodos por medio de la prueba estadística de t-Student. A partir de la prueba de $\mathrm{Chi}^{2}$ se comparó la tasa de esterilidad primaria.

\section{Estimación de los determinantes de la fecundidad Toba}

Para el cálculo de la edad media de la madre al primer hijo, ${ }^{4}$ la media del intervalo entre nacimientos y la edad media al tener el último nacimiento se utilizó toda la muestra de mujeres entrevistadas $(n=336)$. Estos tres determinantes se estimaron a partir del análisis estadístico de supervivencia de Kaplan y Meir ${ }^{5}$ que permite incorporar aquellos casos en los cuales todavía no se produjo el suceso que se pretende observar (casos "censurados" o "truncados"). Para la población de estudio, estos casos corresponden a mujeres en edades reproductivas que no han tenido su primer hijo, a intervalos de nacimientos "abiertos" y a la edad materna al último hijo.

Además, considerando el año de nacimientos de la madre, se analizó la variación temporal de éstos a partir de la regresión de Cox6 (KLEINBAUM, 1996, p. 590).

Todas las pruebas estadísticas antes mencionadas fueron realizadas por medio del paquete informático estadístico SPPS versión 19.0, utilizando una significancia del 5\%.

\section{Resultados}

\section{Estimaciones de la fecundidad}

A partir de un total de 34 mujeres y 219 nacimientos, se obtuvieron los valores de fecundidad correspondientes a las mujeres nacidas en el período 1920-45 (Tabla 1). La variación etaria de la fecundidad muestra una cúspide tardía ubicada entre los 25 y 29 años de edad (Gráfico 1). Para las cohortes de mujeres nacidas entre los años 1946 y 1966, la estructura etaria de fecundidad se obtuvo a partir de un total de 30 mujeres y 227 nacimientos. La distribución de la fecundidad reveló una concentración de la fecundidad a edades menores a los 35 años y un pico de fecundidad aún más tardío, ubicado entre los 30 y 34 años de edad (Gráfico 1).

\footnotetext{
${ }^{4}$ En los grupos indígenas de la región del Gran Chaco el nacimiento del primer hijo no está subordinado a la formación de una pareja estable. Incluso, el embarazo o nacimiento de un hijo puede ser un determinante de la constitución formal de la pareja (IDOYAGA MOLINA, 1999, p. 191). Por lo tanto, no se consideró como un determinante de la fecundidad la edad media al matrimonio. Sólo 17 mujeres respondieron con seguridad con respecto a la edad de la menarca. Por lo que se decidió no considerar este determinante en el análisis.

${ }^{5}$ Es muy común que la variable que se desea estudiar sea el tiempo que tarda en ocurrir un suceso como el nacimiento de un hijo. La característica más importante de este tipo de datos (tiempo hasta que ocurre un suceso) es que al final del período de observación no todos los casos habrán presentado el suceso. Sin embargo, esas observaciones incompletas deben intervenir en el análisis. En la terminología inglesa se les denomina observaciones “censuradas” o "truncadas” y para ello, se utiliza el método de Kaplan-Meier, que se basa en el concepto de probabilidad condicional (KLEINBAUM, 1996, p. 590).

${ }^{6}$ El método de Cox representa otro tipo de modelo de supervivencia en el cual permite analizar la posible relación de la tasa de ocurrencia de un suceso con diferentes variables pronósticos registradas para cada sujeto (KLEINBAUM, 1996, p. 590).
} 
TABLA 1

Variación etaria de la fecundidad específica por edades y la Tasa Global de Fecundidad (TGF) Población Toba Cacique Sombrero Negro - 2003

\begin{tabular}{lrc}
\hline & \multirow{2}{*}{ Edad } & Cohortes \\
\cline { 2 - 3 } & $1920-45$ & $1946-66$ \\
\hline $15-19$ años & 0,0941 & 0,1400 \\
20-24 años & 0,1412 & 0,2400 \\
25-29 años & 0,2588 & 0,2600 \\
30-34 años & 0,2529 & 0,3600 \\
35-39 años & 0,2294 & 0,2533 \\
40-44 años & 0,1882 & 0,1800 \\
45-49 años & 0,1235 & 0,0800 \\
TGF & 6,44 & 7,57 \\
\hline
\end{tabular}

Fuente: Encuestas a mujeres Toba (Proyecto “Cambios demográficos en poblaciones Toba en transición”)

\section{GRÁFICO 1}

Distribución porcentual de la fecundidad del grupo de mujeres nacidas entre 1920 y 1945 y aquellas nacidas en el período 1946-66 Población Toba Cacique Sombrero Negro - 2003

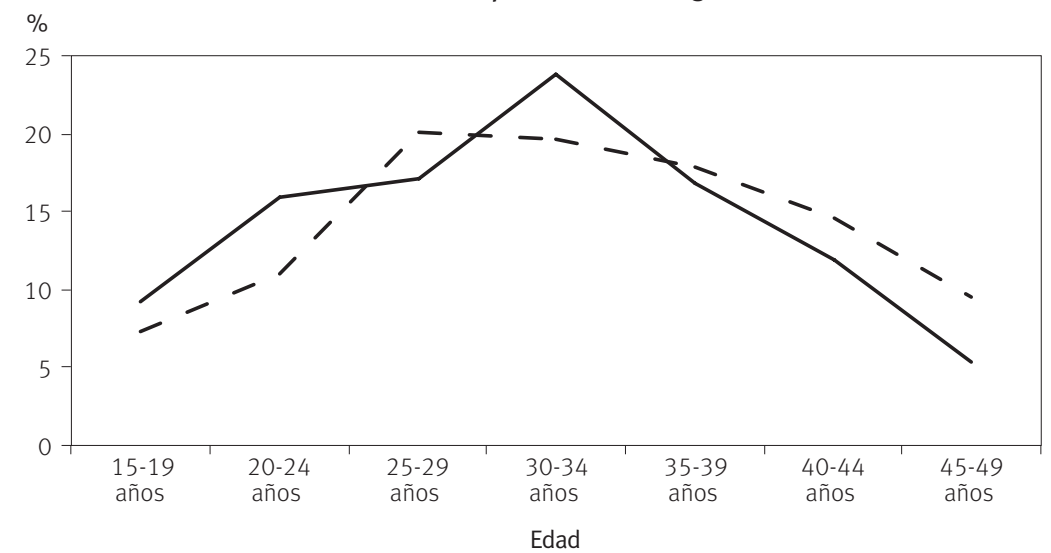

Fuente: Encuestas a mujeres Toba (Proyecto “Cambios demográficos en poblaciones Toba en transición”), 2003.

El aporte de mujeres a la fecundidad total por grupos de edades quinquenales y la edad media de la fecundidad confirman un cambio en la estructura de la fecundidad. El grupo de personas nacidas entre 1946 y 1966 muestra que las mujeres menores a los 35 años contribuyen absoluta y relativamente más a la fecundidad total. La edad media de la fecundidad correspondiente al primer período fue 32,50 años y la del segundo fue 30,93 años, siendo estadísticamente significativa la diferencia $(t=-2,23 ; g l=446 ; p<0,05)$.

A partir de la comparación entre las cohortes, la TGF obtenida mostró un aumento de más de un hijo, siendo ésta de 6,44 hijos para las mujeres pertenecientes a la cohortes 1920-45 y 7,57 hijos para aquellas nacidas entre los años 1946 y 1966 (Tabla 1).

El análisis de la probabilidad de agrandamiento de la familia evidencia en ambas curvas una tendencia cóncava (Gráfico 2). La proporción de mujeres que no tuvieron hijos (esterilidad primaria, 1- $\mathrm{a}_{0}$, Gráfico 2) fue del 8,8\% para las mujeres nacidas entre 1920 y 
1945 y del 6,7\% para aquellas nacidas entre 1946 y 1966 . Sin embargo, las diferencias no fueron estadísticamente significativas $\left(X^{2}=8,75 ; n=1 ; p>0,05\right)$. La curva de probabilidad de agrandamiento de la familia correspondiente al período 1946-66 muestra, en general, valores mayores que aquellos registrados para grupo de mujeres nacidas entre 1920 y 1945 y un aumento de la probabilidad de agrandamiento de la familia entre el sexto hijo $\left(\mathrm{a}_{7}\right)$ y noveno $\left(\mathrm{a}_{10}\right)$ hijo.

\section{GRÁFICO 2}

Variación de la probabilidad de agrandamiento de la familia (an) para mujeres nacidas entre 1920 y 1945 y aquellas nacidas en el período 1946-66

Población Toba Cacique Sombrero Negro - 2003

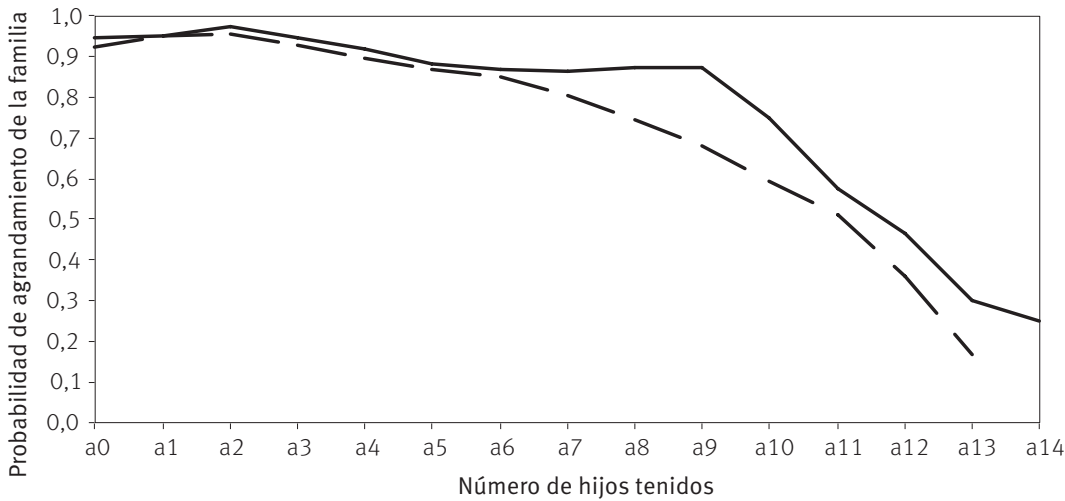

- Mujeres nacidas entre 1920-45 — _ Mujeres nacidas entre 1946-66

Fuente: Encuestas a mujeres Toba (Proyecto “Cambios demográficos en poblaciones Toba en transición”), 2003.

Nota: $a_{n}=$ probabilidad de tener al menos un hijo de orden $n+1$ tras haber tenido un hijo de orden $n$. Por ejemplo, $a_{0}=$ probabilidad de haber tenido por lo menos un hijo, mientras que a1 = probabilidad de tener un segundo hijo habiendo tenido ya uno.

\section{Determinantes de la fecundidad}

A partir de un total de 248 mujeres y de las cuales 7,7\% fueron casos truncados, se obtuvo un valor medio de la edad materna al primer hijo de 20,6 ( \pm 0,41) años y una mediana de 19 $( \pm 0,41)$ años. La variación temporal de este determinante mostró una tendencia temporal descendente y estadísticamente significativa $(B=0,02 ; p<0,01)$.

Con respecto al intervalo entre nacimientos, se obtuvo un valor medio de $28,9( \pm 0,30)$ meses y una mediana de 27,0 $( \pm 0,19)$ meses. Estos valores se obtuvieron a partir de un total de 1253 hijos de los cuales el 10\% fueron truncados. El patrón temporal mostró un descenso en el tiempo siendo éste estadísticamente significativo $(B=0,004 ; p<0,05)$.

La media de la edad materna al último hijo estimada para esta población fue de 43,8 $( \pm 0,64)$ años y la mediana fue de $45( \pm 0,72)$ años. La variación temporal evidenció una tendencia ascendente, siendo esta estadísticamente significativa ( $B=-0,05 ; p<0,01)$. Estos valores fueron obtenidos a partir del total de 278 mujeres, de las cuales el 75,2\% fueron casos censurados. 


\section{Discusión}

Los valores mostrados en este trabajo fueron logrados a partir de métodos retrospectivos y pertenecen a una población pequeña. Por consiguiente, es necesario considerar con prudencia los resultados obtenidos. Además, es posible que, al menos en parte, las tendencias temporales reportadas en este trabajo respondan a una sub-declaración de nacimientos, primordialmente aquellos de primer orden de nacimiento y de hijos fallecidos (BRASS apud NACIONES UNIDAS, 1986, p. 343). En el caso de las poblaciones indígenas, la cautela debe ser aún mayor debido a las diferentes concepciones de tiempo y a los altos niveles de analfabetismo.? No obstante, los niveles y tendencias temporales obtenidos en este trabajo evidencian cierta coherencia resultante, quizás, de complejos procesos socio-económicos y culturales.

La población Toba Cacique Sombrero Negro presenta una variación de la progresión de paridad, en general, típica de poblaciones con un régimen de fecundidad natural (HENRY, 1961, p. 11), una elevada fecundidad y un aumento de las cohortes más jóvenes (1946-66) en comparación a las más ancianas (1920-45). Siendo la elevada paridad un determinante de la mortalidad materna (DONOSO, 2003, p. 55; CHAMY, 2009, p. 331), es posible que la menor fecundidad en el grupo de madres nacidas entre los años 1920 y 1945 responda a una mayor supervivencia de parte de mujeres con un menor número final de hijos. También es factible que este aumento de la fecundidad se deba a un incremento de la mortalidad infantil el cual incrementaría el ritmo reproductivo de las mujeres Toba. No se dispone de datos al respecto para los períodos considerados, sin embargo no es posible descartar un incremento de este determinante por las altas mortalidades testimoniadas por los Toba durante los años de migraciones estacionales a los ingenios azucareros, ocurridas hasta finales de la década de 1960 (GORDILLO, 2006, p. 320).

Por otro lado, es factible que las diferencias de fecundidad respondan a variaciones aleatorias debido al número pequeño de mujeres consideradas. No obstante, las variaciones temporales de los determinantes fueron estimadas a partir de poblaciones mucho mayores y compatibles con un aumento de la fecundidad: un comienzo de la vida reproductiva de las mujeres a edades más tempranas, un mayor ritmo reproductivo y una finalización de su ciclo reproductivo más tardío. Con respecto a la edad materna al primer hijo, es ampliamente aceptado que las mujeres de mayor edad tienden a no declarar sus primeros hijos, especialmente cuando ellos han fallecido a cortas edades (BRASS apud NACIONES UNIDAS, 1986, p. 343). Por lo tanto, es dable que la tendencia descendente de este determinante se deba una sub-declaración de los primeros hijos de las mujeres Toba de mayor edad. Sin embargo, también es posible que los resultados obtenidos respondan a procesos de mayor complejidad. La población aborigen de estudio todavía mantiene aspectos tradicionales de una economía cazadora-recolectora. Aproximadamente el 50\% de los alimentos que

\footnotetext{
${ }^{7}$ Más del 75\% de las mujeres consideradas en este estudio eran analfabetas. Del resto, el número de años formales de estudio fue en promedio de 3 años.
} 
consumen provienen de la actividades de forrajeo (VALEGGIA et al., 2004, p. 19). Autores como Karsten (apud BRAUNSTEIN, 1983, p. 53) dan un papel central a la mujer en la producción y adquisición de recursos en estos grupos aborígenes. Según Mendoza (1999, p. 23), en tiempos históricos las mujeres Toba se dedicaban a la recolección de frutos, tubérculos, rizomas, raíces y semillas entre los meses de setiembre y diciembre. Por su parte, Arenas (2003, p. 562) sostiene que el desarrollo de habilidades y conocimientos para la extracción de recursos de parte de las mujeres era un valor muy apreciado por los hombres para formar pareja y reproducirse. No obstante, la adquisición de las destrezas necesarias para la obtención de recursos del monte puede requerir muchos años. Estimaciones realizadas por Kaplan et al. (2000, p. 29) en poblaciones cazadoras-recolectoras como Ache de Paraguay, Hiwi de Venezuela y Hadza del este de África, muestran que la máxima producción/obtención de recursos se alcanzaba a edades relativamente mayores, variando entre los 20 y 45 años de edad. Estas estimaciones concuerdan con los registros etnográficos relevados por el equipo de trabajo. Por ejemplo, K.P., un hombre Toba de 59 años, explicaba que antes se tenían menos hijos porque los hombres buscaban mujeres más grandes, que ya supieran "arreglarse solas". Ante la pregunta sobre qué edad tenían las mujeres cuando se casaban (en décadas anteriores), K.P. no dudó en decir que eran mujeres mayores a los 20 años. Por lo tanto, y a partir de estos antecedentes, es posible que la edad materna al primer hijo evidencie un descenso temporal.

Por otro lado, es posible que el patrón de variación de este determinante sea el resultado del abandono de normas y comportamientos tradicionales. En los Toba, al igual que en otras etnias de la región, eran habituales las prácticas abortivas (IDOYAGA MOLINA, 1999, p. 191). Su ejercicio se realizaba fundamentalmente en etapas en las cuales las jóvenes adolescentes gozaban de cierta libertad sexual pre-matrimonial. Si la actividad sexual juvenil resultaba en un embarazo, existía la alternativa de interrumpir la gestación (IDOYAGA MOLINA, 1999, p. 191). Sin embargo, las presiones ejercidas por diferentes religiones cristianas y el Estado Nacional habrían logrado que esta práctica haya desaparecido o, por lo menos, haya disminuido sustancialmente (VITAR, 1999, p. 23). Como consecuencia, es posible que el descenso de la edad materna al primer hijo resulte de un abandono de prácticas abortivas en adolescentes.

El infanticidio era otra de las prácticas ejercidas por estos grupos (IDOYAGA MOLINA, 1999, p. 191). Podía realizarse, al igual que el aborto, como consecuencia de un embarazo no deseado durante las etapas de libertad sexual adolescente, por el nacimiento de mellizos o de niños con algún tipo de anormalidad morfológica (IDOYAGA MOLINA, 1999, p. 191). Su ejercicio también fue duramente sancionado por instituciones oficiales y religiosas, por lo que habría desaparecido. De esta forma, no es posible descartar que la disminución de la edad materna al primer hijo obtenida en este trabajo no fuera real y, a medida que la práctica del infanticidio iba desapareciendo, se produce una mayor declaración de nacimientos de niños antiguamente no deseados por sus padres.

En poblaciones pre-transicionales un importante determinante del ritmo reproductivo está determinado por el tiempo transcurrido del retorno de la fertilidad luego del parto 
(BONGAARTS, 1978, p. 28; WOOD, 1994, p. 653). Al mismo tiempo, existen numerosos antecedentes indicando que la condición física de la madre antes y durante la gestación y amamantamiento de su hijo determina la rapidez de su recuperación física que le permita engendrar un nuevo descendiente (VALEGGIA; ELLISON, 2003, p. 22). La población Toba de estudio se encuentra en una transición nutricional incrementándose la prevalencia de obesidad y sobrepeso (VALEGGIA et al., 2010, p. 100). De esta forma, se puede hipotetizar que las mujeres Toba tendrían una mayor disponibilidad de energía metabólica que les permita un más rápido retorno de la fertilidad luego del parto. Como consecuencia, puede predecirse una disminución del intervalo entre nacimientos. También un menor tiempo de amamantamiento puede determinar un acortamiento del período de infertilidad post-parto (WOOD, 1994, p. 653) disminuyendo el intervalo entre nacimientos. Resultados obtenidos por Lanza (2009, p. 260) sugieren que no es posible descartar esta posibilidad.

Con relación a la edad materna al último nacimiento, existe acuerdo que las mujeres en edades avanzadas de su ciclo reproductivo presentan una mayor tasa de mortalidad materna (DONOSO, 2003, p. 55; CHAMY, 2009, p. 331). Por lo tanto, es posible suponer que ciertas mejoras de las condiciones de vida de la población Toba hayan determinado una disminución de la mortalidad materna correspondiente a mujeres con una elevada paridez determinando, como resultado, un aumento temporal de este determinante. Resultados obtenidos por Lanza y Valeggia (2013b, en prensa) en la misma población de estudio apoyarían este concepto. A partir de la distribución etaria de muertes ocurridas en los períodos 1980-94 y 1995-2010, los autores muestran una mayor proporción de fallecimientos masculinos en relación a los femeninos entre los 30 y 44 años, posiblemente debido a una menor mortalidad materna. Sin embargo, también es factible que este aumento de la edad materna al último hijo responda a una mayor supervivencia masculina en etapas reproductivas. Una menor mortalidad de hombres determinaría que las mujeres permanezcan unidas por un mayor tiempo extendiendo su vida reproductiva y, por lo tanto, causando un aumento de la edad al último hijo. Investigaciones realizadas por Lanza y Valeggia (2013a, p. 212) en la misma población indígena comparan datos censales de los años 1985 y 2002 evidenciando un incremento de la razón de sexos entre los 35 y 54 años en el segundo censo apoyando la hipótesis anterior. Aunque estos resultados también podrían obedecer a procesos de migración, situación que los autores consideran improbable, también es posible que se deba a una mayor supervivencia masculina.

Por otro lado, la disminución de la fecundidad en países latinoamericanos ha sido asignado a mejoras en las condiciones de vida de la población (SCHKOLNIK, 2004, p. 23), determinado, entre otros factores, por un mejoramiento económico de la población. Incluso, autores como Bongaarts y Watkins (1996, p. 639) y Caldwell (2006, p. 426) definen al crecimiento económico como un pre-requisito esencial para el descenso de la fecundidad. La población Toba presentaría cierto grado de crecimiento económico pero con ciertas particularidades. En la población de estudio, hasta el año 2004 el 50\% de los alimentos consumidos provenían de su economía tradicional (VALEGGIA et al., 2004, p. 123). Sin 
embargo, desde finales del siglo XIX éstas no fueron sus únicas actividades económicas. Desde esos años, y hasta finales de la década de 1960, los Toba de la población Cacique Sombrero Negro comenzaron a insertarse en una economía capitalista a partir de la venta estacional de su mano de obra en los ingenios azucareros y en las cosechas de porotos y de algodón. Conjuntamente, en la década de 1930 comenzó a desarrollarse la agricultura de subsistencia y, hasta la actualidad, la producción mercantil de artesanías realizadas principalmente por las mujeres (GORDILLO, 1996, p. 135). Asimismo, desde comienzos de la década de 1980 el trabajo asalariado se acrecienta a partir una gran distribución de empleo público entre los hombres. Esto permitió a los Toba obtener cargos administrativos en el estado municipal y provincial (GORDILLO, 2002, p. 262) y ocupar puestos en el sistema de salud y educativo. Igualmente, desde esos mismos años, también entre las mujeres se produce una creciente entrega de subsidios y pensiones sociales (GORDILLO, 2006, p. 320). En este sentido, es interesante lo registrado por Gordillo (2006, p. 320) quien resalta los conflictos que se producen en esta misma población Toba a partir de una valoración de las bondades de una economía de mercado y aquellas propias de una economía forrajeadora. El mismo autor observa que durante los períodos de crisis económica de la Argentina, los Toba realizan un mayor aprovechamiento de los alimentos de extracción. "En el monte tenemos todo para vivir" expresaba un Toba durante las entrevistas realizadas por nuestro grupo de trabajo. Es decir, desde finales del siglo XIX se producía una convivencia de diferentes sistemas productivos definida por la persistencia de actividades económicas tradicionales de forrajeo y una mayor inserción en la economía capitalista. Sin embargo, estas condiciones particulares de crecimiento económico habrían determinado, en vez de una disminución de la fecundidad, un aumento de ésta.

Para el caso de las poblaciones nómadas, los cambios en su estilo de vida incluye también implican el proceso de sedentarización el cual es ampliamente asociado a un incremento de la fecundidad (MEIR, 1986, p. 199; JOSEPH, 2004, p. 140; SUEYOSHI; OHTSUKA, 2008, p. 1). Para el caso de la población de estudio, este proceso se inició en el año 1930. Según Mendoza (2002, p. 235), en esos años los Toba se encontraban en una situación de gran adversidad determinada por los constantes conflictos con los colonos que ocupaban sus tierras y los primeros enfrentamientos armados entre Bolivia y Paraguay. Esto obligó a los Toba a recurrir a las misiones anglicanas en busca de medicamentos y a pedir que actuaran como mediadores en sus litigios con los colonos que ocupaban sus tierras. De esta forma, en 1930 los pastores Alfred Leake y Alec Sanderson fundan la misión El Toba en la comunidad Sombrero Negro, centro de las tierras Toba. Esto produjo una concentración de muchas de las bandas alrededor de la misión religiosa, un incremento del intercambio de bienes, un mayor acceso de los Toba a la atención médica a iniciar un proceso de alfabetización por parte de los misioneros. Esta situación determinó que muchas de las bandas, ${ }^{8}$ aunque no todas, se asentaran en forma definitiva en las cercanías de ésta. Otro evento ocurrió en el año 1989 en

\footnotetext{
$\overline{8}$ La organización social de las poblaciones indígenas del Gran Chaco están conformadas por bandas las cuales son definida como un conjunto de familias emparentadas por medios vínculos matrimoniales (MENDOZA, 2002, p. 235).
} 
el cual el Estado Provincial les entregó 35.000 has. de tierra como parte del reconocimiento de sus reclamos. Este importante hecho significó la culminación del proceso de sedentarización produciendo el asentamiento definitivo de la población Cacique Sombrero Negro.

Por otro lado, Mendoza (2002, p. 235) sostiene que el proceso de sedentarización a comienzos del siglo XX fue determinado por el acceso a medicamentos demandados a partir de las epidemias de la gripe y sarampión que se estaban produciendo. En relación a la fecundidad, Frank (1983, p. 137) observa que a partir de la utilización en la década de 1950 de la penicilina contra la sífilis se registró un incremento de la fecundidad en países africanos como Camerún, Zaire y Zambia. Pagliaro (2002, p. 194) realiza similares especulaciones para explicar el aumento de la fecundidad en una población Kaiabi de Brasil. Para el caso de la población Toba de estudio, no se disponen de datos que permitan evaluar la prevalencia, y por esto el impacto sobre la fecundidad, de enfermedades de transmisión sexual como las mencionadas. Sin embargo, Bargalló (1992, p. 128) muestra que durante la década de 1960 se iniciaron diferentes programas de vacunación y se construyeron en la región centros médicos de mediana y baja complejidad. En consecuencia, es factible que esta situación determinara una menor prevalencia de enfermedades infecto-contagiosas produciendo un incremento de la fecundidad y una reducción en la prevalencia de esterilidad primaria en la población Toba, registrada en esta investigación (aunque las diferencias no fueron estadísticamente significativas).

Finalmente, los cambios de estilo de vida de la población de estudio pueden implicar el debilitamiento de normas culturales que regulan la fecundidad (NAG, 1980, p. 571; RODRÍGUEZ VIGNOLI, 2004, p. 93). En este sentido, es interesante la distinción realizada por Caldwell (1976, p. 321) quien define "occidentalización" como la adopción de normas culturales, sociales y económicas de la sociedad occidental, caracterizada por los bajos niveles de fecundidad. En consecuencia, el descenso de la fecundidad sería el resultado de la incorporación de normas comportamentales que se practican en "occidente" como por ejemplo la aceptación de métodos anticonceptivos y educación formal de parte del estado y una mayor participación femenina en el campo laboral. Sin embargo, es interesante destacar el matiz que se produce para caso de la población Toba Cacique Sombrero Negro. En ésta, la occidentalización consiste del abandono de pautas comportamentales y tradicionales las cuales no se practican en la sociedades “occidentales”. Entonces, como consecuencia de este socavamiento cultural, es posible hipotetizar que el aumento de la fecundidad registrado en este trabajo responda a una devaluación de las mujeres socialmente valoradas para la reproducción, fundamentalmente aquellas "que se las rebuscan solas en el monte" disminuyendo la edad materna al primer nacimiento (IDOYAGA MOLINA, 1999, p. 191; ARENAS, 2003, p. 562; LANZA et al., 2011, p. 47), y al abandono de prácticas que extienden el intervalo intergenésico (HUFFMAN et al., 1980, p. 144; ROMANIUK, 1981, p. 157; TOLA, 1998, p. 27).

Por un último, un aspecto a considerar es la elevada edad modal de la curva de fecundidad del grupo de mujeres nacidas entre 1945 y 1966. Es posible que este patrón responda a un sub-declaración de nacimientos correspondiente a estas cohortes de madres. 
Sin embargo, esto implicaría un menor registro de nacimientos del grupo de mujeres más jóvenes en relación a las más ancianas, situación poco probable. Finalmente, también es posible que el pico de fecundidad entre 30 y 34 años responda a una sobrestimación en la edad materna de nacimiento en relación a los ocurridos entre los 25 y 29 años, el cual evidencia un descenso relativo.

Sin embargo, investigaciones realizadas en poblaciones Ache de Paraguay (HILL; HURTADO, 1996, p. 561), Hupd'ah de Brasil (MACHADO et al., 2009, p. 14) y Gabbra de Kenya (MACE; SEAR, 1996, p. 5) obtuvieron también una edad modal en el segmento etario 30-34. Por otro lado, Lanza et al. (2013a, p. 212) muestran, para la misma población, una variación temporal de la curva de fecundidad caracterizada por un aumento de la edad modal entre los años 1981 y 1994 registrándose un pico entre los 30 y 34 años de edad materna en el período 1990-1994.

\section{Conclusión}

Según Zavala de Cosío (1992, p. 11), los cambios de la fecundidad responden a variaciones de diferentes factores ecológicos de la población. Esto plantea la necesidad de reconocer los diferentes contextos históricos, sociales, culturales y económicos en los cuales los individuos se reproducen. En el caso de las poblaciones indígenas, la necesidad de generar políticas “culturalmente pertinentes" implica la necesidad de distinguir de qué manera sus particularidades culturales, históricas, sociales y económicas afectan su dinámica demográfica. Los resultados expuestos en este trabajo sugieren que las variaciones en el estilo de vida de esta población provocaron un aumento de la fecundidad. Para la población Cacique Sombrero Negro, este cambio estaría definido por cambios sociales (valoración sobre las mujeres en capacidad de constituir una pareja), sanitarios (mayor supervivencia de las madres y/o esposos), biológicos (una menor infertilidad post-parto) y económicos (cierta inserción en la economía capitalista y la persistencia de cierta dinámica tradicional de su economía).

Por otro lado, los cambios en el estilo de vida de la población de estudio también habrían determinado un debilitamiento de normas que regulaban la fecundidad. A la luz de los resultados aquí expuestos esta situación produce, aún en la actualidad (LANZA et al., 2011, p. 47), un "vacío" de prácticas alternativas que permitan controlar la fecundidad.

En resumen, considerar la variedad de los contextos en que se desarrollaron los cambios demográficos permitirá elaborar modelos demográficos que integren estos grupos históricamente marginados, generar políticas acordes a su realidad socio-cultural y comprender más profundamente por qué cambió la fecundidad en aquellos contextos.

\section{Referencias}

ARCE BORDON, A. Población indígena. Asunción: DGEEC, 2005.

ARENAS, P. Etnografía y alimentación entre los Toba-Ñachilamole\#ek y Wichí-Lhuku’tas del Chaco Central (Argentina). Buenos Aires: Pastor Arenas, 2003. 
BANCO MUNDIAL. Primer Proyecto de Préstamo Adaptable para el Proyecto de Seguro de Salud Materno Infantil Provincial: Plan para los pueblos indígenas (v. 3 of 8); Plan de pueblos indígenas 2010; Plan Nacer Provincia de Formosa. Argentina, 2010.

BARGALLÓ, M. Shamanes, iglesias y atención primaria entre los tobas del oeste de Formosa. Etnicidad y hegemonización en el campo de la salud. Tesis de grado. Buenos Aires: Universidad de Buenos Aires, 1992.

BAY, G.; MACADAR, D. Boletín Demográfico. América Latina: Fecundidad 1950-2050. Santiago de Chile: Celade-Cepal, 2001.

BENTLEY, G.; JASIENSKA, G.; GOLDBERG, T. The fertility of agricultural and non-agricultural traditional societies. Population Studies, v. 47, n. 2, p. 269-28, 1993.

BONGAARTS, J. A framework for analyzing the proximate determinants of fertility. Population and Development Review, v. 4, n. 1, p. 105-132, 1978.

BONGAARTS, J.; WATKINS, S. Social interactions and contemporary fertility transitions. Population and Development Review, v. 22, n. 4, p. 639-682, 1996.

BRAUNSTEIN, J.; MILLER, E. Ethnohistorical introduction. In: MILLER, E. (Org.). Peoples of the Gran Chaco. Westport, CT: Bergin \& Garvey, 1999, p. 1-22.

CALDWELL, J. Toward a restatement of demographic transition theory. Population and Development Review, v. 2, n. 3/4, p. 321-366, 1976.

Demographic transition theory. Dordrecht: Springer, 2006.

CEPAL-CELADE. La fecundidad en América Latina: ¿transición o revolución? Santiago de Chile: CepalCelade, 2004

CHACKIEL, J. La transición de la fecundidad en América Latina 1950-2000. Papeles de Población, v. 41, p. 9-58, 2004.

CHACKIEL, J.; SCHKOLNIK, S. América Latina: los sectores rezagados en la transición de la fecundidad. In: CEPAL-CELADE (Orgs.). La fecundidad en América Latina: ¿transición o revolución? Santiago de Chile: Cepal-Celade, 2004, p. 51-74.

CHAMY, V.; CARDEMIL, F.; BETANCOUR, P.; RÍOS, M.; LEIGHTON, L. Riesgo obstétrico y perinatal en embarazadas mayores de 35 años. Revista Chilena de Obstetricia y Ginecología, v. 74, n. 6, p. 331-338, 2009.

DEL POPOLO, F.; SCHKOLNIK, S.; JASPERS FAIJER, D. Los pueblo indígenas de Bolivia: diagnóstico socio-demográfico a partir del censo del 2001. Santiago de Chile: Cepal, 2005.

DONOSO, E.; VILLARROEL, L. Edad materna avanzada y riesgo reproductivo. Revista Médica de Chile, V. 131 , n. 1, p. 55-59, 2003

FRANK, O. Infertility in sub-Saharan Africa: estimates and implications. Population and Development Review, v. 9, n. 1, p. 137-144, 1983.

GOLDBERG, C. Reflexiones en torno a la medición de la etnicidad en censos y encuestas. In: IX JORNADAS ARGENTINAS DE ESTUDIOS DE POBLACIÓN, HUERTA GRANDE. Córdoba, 2007.

GORDILLO, G. Entre el monte y las cosechas: migraciones estacionales y retención de fuerza de trabajo entre los Tobas del oeste de Formosa (Argentina). Estudios Migratorios Latinoamericanos, v. 11, n. 32, p. 135-167, 1996.

. Locations of hegemony: the making of places in the Toba's struggle for La Comuna, 1989-99.

American Anthropologist, v. 104, n. 1, p. 262-277, 2002. 
En el Gran Chaco. Buenos Aires: Prometeo, 2006.

HENRY, L. Some data on natural fertility. Eugenics Quarterly, v. 8, p. 81-91, 1961.

HILL, K.; HURTADO, A. Ache life history. The ecology of a foraging people. New York: Aldine de Gruyter, 1996.

HIRSCHMAN, C. Why fertility changes. Annual Review of Sociology, v. 20, p. 203-233, 1994.

HUFFMAN, S.; CHOWDHURY, A.; CHAKRABORTY, J.; SIMPSON, N. Breast-feeding patterns in rural Bangladesh. American Journal of Clinical Nutrition, v. 33, p. 144-154, 1980.

IDOYAGA MOLINA, A. Sexualidad, reproducción y aborto. Buenos Aires: Caea-Conicet, 1999.

JOSEPH, S. The biocultural context of very high fertility among the Bekaa Bedouin. American Anthropologist, v. 106, n. 1, p. 140-144, 2004.

KLEINBAUM, D. Survival analysis. New York: Springer-Verlag, 1996.

LANZA, N. Análisis del comportamiento reproductivo de una población Toba del Oeste Formoseño. Tesis Doctoral. Córdoba: Universidad Nacional de Córdoba, 2009.

LANZA, N.; VALEGGIA, C.; PELAÉZ, E. Análisis de la fecundidad de los Toba del norte de Argentina. In: DEL POPOLO, F.; GARCIA DE PINTO DA CUNHA, E.; RIBOTTA, B.; AZEVEDO, M. (Orgs.). Pueblos indígenas y afrodescendientes en América Latina: dinámicas poblacionales diversas y desafíos comunes. Montevideo: Trilce, p. 47-72, 2011.

LANZA, N.; VALEGGIA, C. La modernización, ¿siempre disminuye la fecundidad? Análisis de la transición demográfica en una población Toba. Revista Argentina de Antropología Biológica, v. 14, n. 1, p. 33-43, 2012.

Cambios demográficos en una población rural de la etnia Toba del norte de Argentina.

Latin American Research Review, v. 49, n. 2, p. 212-230, 2013 a.

Modernización, mortalidad y salud de una población rural Toba de Formosa, Argentina. In: MEICHTRY N.; MUSCAR BENASAYAG, E. (Orgs.). Vulnerabilidad, inclusión y fragmentación socioespacial en América Latina. Barcelona: Universidad Nacional Complutense, 2013b (en prensa).

LANZA, N.; VALEGGIA, C.; PELÁEZ, E. The reproductive transition in an indigenous population of northern Argentina. Biodemography and Social Biology, v. 59, n. 2, p. 212-230, 2013.

MEIR, A. Demographic transition theory: a neglected aspect of the nomadism-sedentarism continuum. Transactions of the Institute of British Geographers, v. 11, n. 2, p. 199-211, 1986.

MELIÀ, B. Pueblos indígenas en el Paraguay. Asunción: DGEEC, 1997.

MENDOZA, M. Band mobility and leadership among western Toba Hunter-Gatherers of Gran Chaco in Argentina. Lewiston\Queenston\Lampeter: The Edwin Mellen Press, 2002.

MUNILLA, D.; GOLDZTEIN, N. El Censo argentino entre dos milenios. Presentación de resultados post SEPOSAL 2000. Los pueblos indígenas en la argentina a través del censo 2001. Salta - Argentina, 2005.

NACIONES UNIDAS. Manual X. Técnicas indirectas de estimación demográfica. Nueva York: Naciones Unidas, 1986.

NAG, M. How modernization can also increase fertility? Current Anthropology, v. 21, n. 5, p. 571-587, 1980.

PAGLIARO, H. A revolução demográfica dos povos indígenas do Brasil: a experiência dos Kaiabi do Parque Indígena do Xingu - Mato Grosso-1970-1999. Tese (Doutorado). São Paulo: Universidade de São Paulo, 2002 
PAGLIARO, H.; JUNQUEIRA, C. Recuperação populacional e fecundidade dos Kamaiurá, povo Tupi do alto Xingu, Brasil Central, 1970-2003. Cadernos de Saúde Pública, v. 16, n. 2, p. 37-47, 2007.

POPKIN, B. An overview on the nutrition transition and its health implications: the Bellagio meeting. Public Health Nutrition, v. 5, n. 1a, p. 93-103, 2002.

RODRÍGUEZ VIGNOLI, J. La fecundidad alta en América Latina y el Caribe: un riesgo en transición. In: CEPAL-CELADE (Orgs.). La fecundidad en América Latina: ¿transición o revolución? Santiago de Chile: Cepal-Celade, 2004, p. 93-140.

ROMANIUK, A. Increase in natural fertility during the early stages of modernization: Canadian indians case study. Demography, v. 18, n. 2, p. 157-172, 1981.

SCHKOLNIK, S. La fecundidad en América Latina. In: CEPAL-CELADE (Orgs.). La fecundidad en América Latina: ¿transición o revolución? Santiago de Chile: Cepal-Celade, 2004, p. 33-48.

SKINNER, G. Family systems and demographic processes. In: KERTZER, D.; FRICKE, T. (Orgs.). Anthropological demography. Toward a new synthesis. Chicago \London: The University of Chicago Press, 1997, p. 53-95.

SUEYOSHI, S.; OHTSUKA, R. Extremely high fertility of a sedentarized Bedouin clan in south Jordan: a genealogical-demographic approach to long-term change. Anthropological Science, v. 116, n. 1, p. 1-8, 2008.

TOLA, F. La restricción sexual en la lactancia y la "lucha entre hermanos" en un grupo Toba de Formosa. Anales de la Sociedad Científica, v. 228, n. 2, p. 27-37, 1998.

TORRADO, S. Transición de la familia en la Argentina, 1870-1995. Desarrollo Económico, v. 39, n. 154 , p. 235-260, 1999.

VALEGGIA, C.; LANZA, N.; CÓRDOBA, L. Fuentes de variación en la alimentación actual de los Toba-Pilagá del oeste formoseño. Anales de la Sociedad Argentina de Americanistas. Buenos Aires, 2004, p. 123-142.

VALEGGIA, C.; BURKE, K.; FERNANDEZ DUQUE, E. Nutritional status and socioeconomic change among Toba and Wichi populations of the Argentine an Chaco. Economics and Human Biology, v. 8, n. 1, p. 100-110, 2010.

WOOD, J. Dynamics of human reproduction. Biology, biometry, demography. New York: Aldine de Gruyter, 1994.

ZAVALA DE COSíO, M. La transición demográfica en América Latina y Europa. Notas de Población, v. 20, n. 56, p. 11-32, 1992.

\section{Autores}

Norberto Lanza es doctor en Biología, Investigador Asistente del Consejo Nacional de Investigaciones Científicas y Tecnológicas de la República Argentina.

Claudia Valeggia es doctora en Comportamiento Animal, Profesora Asociada del Departamento de Antropología de la Universidad de Pennsylvania, Estados Unidos.

\section{Dirección para la correspondencia}

Norberto Lanza

Av. Castelli 930

Resistencia, Chaco, Argentina - CP 3500 


\section{Resumo}

Análise longitudinal do comportamento reprodutivo de uma população Toba em transição

As mudanças demográficas na população, muitas vezes, respondem às mudanças ambientais. Portanto, as características sociais, econômicas e culturais das populações indígenas produzem uma variedade de regimes demográficos. Enquanto na América Latina, em geral, e na Argentina, em particular, registrou-se um declínio significativo da fecundidade, as populações indígenas apresentam mudanças demográficas que diferem dessa tendência. Este artigo analisa os determinantes da fecundidade e da população Toba Cacique Black Hat, do norte da Argentina, por meio dos quais ocorreram mudanças em seu estilo de vida. Foram entrevistadas 336 mulheres, calculando-se as taxas de fecundidade, a probabilidade de uma família ampliada e a taxa de esterilidade primária para mulheres nascidas entre 1920 e 1966. Estimaram-se idade materna no primeiro parto, intervalo de nascimento e idade da mãe no último filho. Foram registrados aumento na fecundidade, diminuição na idade materna no primeiro filho, taxa de reprodução mais elevada e um aumento da idade materna no último filho na coorte de nascidas entre 1946 e 1966. Os resultados sugerem mudanças sociais, de saúde, biológicas e econômicas favoráveis, que têm proporcionado o aumento da fecundidade em coortes mais jovens.

Palavras-chave: Toba. Fecundidade. Determinantes da fecundidade. Transição demográfica.

\section{Abstract}

Longitudinal analysis of reproductive behavior of a Toba transition population

Demographic variations in a given population are frequently associated to environmental changes. The social, economic, and cultural particularities of indigenous populations would then produce a great variety of demographic regimes. Although Latin America, in general, and in Argentina, in particular, have experienced an important decline in fertility, indigenous populations in this region show demographic changes that are at odds with this trend. This study evaluates fertility parameters and their determinants in the Toba population of Cacique Sombrero Negro, in northern Argentina, which is undergoing a significant lifestyle change. A total of 336 Toba women were interviewed, allowing the calculation of age-specific fertility rates, total fertility rates, parity progression ratios and primary infertility rates for women born between 1920 and 1966. For these women, we also estimated age of the mother at first birth, interbirth intervals and mother's age at last birth. Our results indicate that, compared to women born between 1920 and 1945, those born between 1946 y 1966 show an increase in fertility values, a decline in age at first birth, a faster reproductive pace and an increase in age at last birth. These results suggest favorable social, sanitary, biological and economic changes that would have determined an increase in fertility parameters in the younger cohort.

Keywords: Toba. Fertility. Fertility determinants. Demographic transition.

Recebido para publicação em 05/09/2012

Aceito para publicação em 06/04/2014 